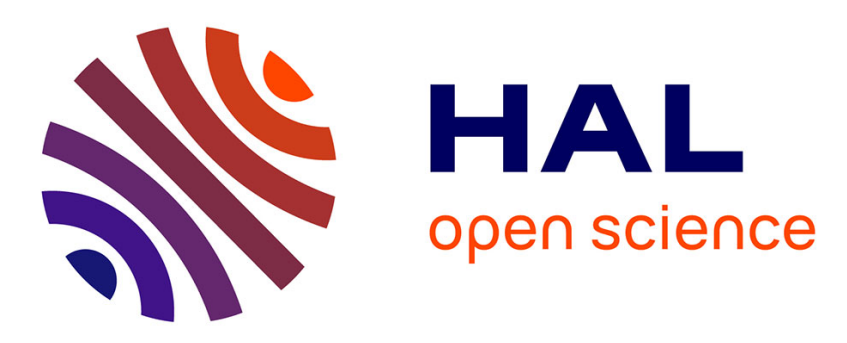

\title{
Altered vaccine-induced immunity in children with Dravet syndrome
}

Stéphane Auvin, Mohamed Jeljeli, Béatrice Desnous, Nadia Soussi-Yanicostas, Pascal Dournaud, Ghislaine Sterkers

\section{- To cite this version:}

Stéphane Auvin, Mohamed Jeljeli, Béatrice Desnous, Nadia Soussi-Yanicostas, Pascal Dournaud, et al. Altered vaccine-induced immunity in children with Dravet syndrome. Epilepsia, 2018, 59 (4), pp.e45-e50. 10.1111/epi.14038 . hal-02357179

\section{HAL Id: hal-02357179 \\ https://hal.science/hal-02357179}

Submitted on 9 Nov 2019

HAL is a multi-disciplinary open access archive for the deposit and dissemination of scientific research documents, whether they are published or not. The documents may come from teaching and research institutions in France or abroad, or from public or private research centers.
L'archive ouverte pluridisciplinaire HAL, est destinée au dépôt et à la diffusion de documents scientifiques de niveau recherche, publiés ou non, émanant des établissements d'enseignement et de recherche français ou étrangers, des laboratoires publics ou privés. 


\title{
Altered vaccine-induced immunity in children with Dravet syndrome
}

\author{
Stéphane Auvin',2 (D) | Mohamed Jeljeli ${ }^{3}$ | Béatrice Desnous ${ }^{1,2}$ | Nadia Soussi-Yanicostas ${ }^{1}$ | \\ Pascal Dournaud $^{1}$ | Ghislaine Sterkers ${ }^{3}$
}

${ }^{1}$ PROTECT, INSERM, Paris Diderot University, Sorbonne Paris Cité, Paris, France

${ }^{2}$ APHP, Pediatric Neurology Department, Robert-Debré University Hospital, Paris, France

${ }^{3}$ APHP, Clinical Immunology

Department, Robert-Debré University

Hospital, Paris, France

\section{Correspondence}

Stéphane Auvin, Service de Neurologie Pédiatrique et des Maladies Métaboliques, CHU Hôpital Robert Debré, Paris Cedex

19, France.

Email: stephane.auvin@aphp.fr

Funding information

INSERM; Université Paris-Diderot;

Fondation de l'Avenir

\begin{abstract}
Summary
Dravet syndrome (DS) is a refractory epileptic syndrome. Vaccination is the trigger of the first seizure in about $50 \%$ of cases. Fever remains a trigger of seizures during the course of the disease. We compared ex vivo cytokine responses to a combined aluminium-adjuvanted vaccine of children with DS to sex- and agematched heathy children. Using ex vivo cytokine responses of peripheral-blood mononuclear cells and monocytes, we found that vaccine responsiveness is biased toward a proinflammatory profile in DS with a M1 phenotype of monocytes. We provide new insight into immune mechanisms associated with DS that might guide research for the development of new immunotherapeutic agents in this epilepsy syndrome.
\end{abstract}

\section{K E Y W O R D S}

cytokines, Dravet syndrome, epilepsy, inflammation, monocytes

\section{1 | INTRODUCTION}

Dravet syndrome (DS) is a refractory epileptic syndrome starting before the age of 1 . The first seizure is typically a febrile status epilepticus. Shortly thereafter other seizures occur leading to diagnosis in an otherwise normal infant. Later, myoclonus, atypical absences, and partial seizures are observed. Developmental delay becomes apparent within the second year of life. Mutations in the voltagegated sodium channel gene SCN1A are the cause of DS in $70 \%-80 \%$ of the patients. Fever is the most common trigger of seizures during the course of the disease. ${ }^{1,2}$ Vaccination is the trigger of the first seizure in about $50 \%$ of cases. ${ }^{3,4}$ However, precipitation of seizure onset by immunization does not affect the course of the disease. ${ }^{5}$

A growing body of evidence implicates a link between inflammation and epileptic disorders. ${ }^{6,7}$ Most studies analyzed the baseline levels of proinflammatory cytokines in tissue, sera, or cerebrospinal fluid (CSF). ${ }^{6,8} \mathrm{~A}$ restricted number of studies evaluated cytokine levels following ex vivo stimulation of immune cells with nonspecific activators. ${ }^{9}$ Ex vivo responses to vaccine antigens reflecting in vivo priming with vaccines avoid seizure-induced cofounding factors. Despite evidence for vaccine-related seizure onset in DS, nothing is known about cytokine response to vaccines in these patients. In DS, experimental studies have shown that the SCN1A mutation results in a modified neuronal excitability, which is sensitive to the increase in body temperature. ${ }^{10}$ Whether inflammation contributes to seizure occurrence has not yet been investigated. Here, we explored if vaccinated patients with DS might be prone to an altered cytokine response to the vaccine.

\section{2 | POPULATIONS AND METHODS}

We compared ex vivo cytokine responses to a combined aluminium-adjuvanted vaccine (diphtheria-tetanus-pertussispoliomyelitis-haemophilus-B hepatitis; Infanrix-hexa, GlaxoSmithKline Biologicals, Brentford, UK) of children 
with DS (Table S1) to sex- and age-matched heathy children (HC). Inclusion criteria were completion of vaccinations and availability of residual cryopreserved peripheral-blood mononuclear cells (PBMCs) from blood samples collected for standard care in stable clinical conditions (DS) at least 15 days from any fever/infection episode or for biological investigations in healthy bone marrow donors before the gift (HC). The study was approved by our hospital's institutional review board (Robert-Debré Hospital, Paris, France). Informed consent was obtained from the parents of all children. Table $\mathrm{S} 2$ reports the main characteristics of DS.

Cryopreserved PBMCs from DS and HC were simultaneously thawed and seeded $\left(10^{6} / \mathrm{mL}\right)$ into Roswell Park Memorial Institute culture medium supplemented with pooled human sera (10\%), glutamine (1\%), and antibiotics. Cell suspensions were cultured at $37^{\circ} \mathrm{C}$, in $5 \% \mathrm{CO}_{2}$ atmosphere, overnight with the vaccine at a predetermined optimal dilution $(10 \%)$ or left unstimulated. Cytokines were measured blindly in the supernatants by a multiplexed-cytokine assay using the 17-Plex human kit (Bio-Rad, Laboratories Inc., Marne-La-coquette, France) as described previously. ${ }^{11}$ Supernatants from the 2 culture conditions were analyzed for each child. Sufficient amounts of PBMCs were available to isolate monocytes in 5 DS patients and their respective controls. PBMCs $\left(2 \times 10^{6}\right)$ from each individual were distributed into culture medium $(1 \mathrm{~mL})$ supplemented with $10 \%$ fetal calf serum, and monocytes were allowed to adhere to plastic by incubation of the cell suspension in flat-bottom culture walls for 2 hours at $37^{\circ} \mathrm{C}$. After careful removal of nonadherent cells, culture medium was added $(1 \mathrm{~mL})$ to monocytes and cultures were processed as described earlier for PBMCs.

Data were analyzed using the Prism 5 software (GraphPad, San Diego, CA, USA). Cytokine/chemokine releases are expressed as median and range in Table 1. Vaccinespecific cytokine/chemokine releases are expressed as mean \pm standard error of the mean (SEM) in Figure 1. Statistical analysis was performed using Mann-Whitney test. $P$ values are considered statistically significant when $P<.05$.

\section{3 | RESULTS}

We exclude 5 cytokines/chemokines showing either concentrations lying outside an interpretable range (interleukin8 [IL-8], monocyte chemoattractant protein-1 [MCP-1], macrophage inflammatory protein 1-beta [MIP1- $\beta]$ ) or similar levels in all culture conditions (IL-7, IL-12).

As shown in Table 1, the median levels of cytokine release in the supernatants from unstimulated PBMCs (background) were similar in DS and HC. In vitro stimulation with the vaccine induced in both groups a marked increase, over background, in the secretion of Type 1 T helper (Th1) (IL-2, IFN- $\gamma$ ), Th2 (IL-4, IL-13), and Th17 (IL-17) T cell-derived cytokines, as well as in the proinflammatory (IL-1 $\beta$, IL-6, tumor necrosis factor alpha [TNF- $\alpha]$, granulocyte-colony stimulating factor [GCSF], granulocytemacrophage colony-stimulating factor [GMCSF], MIP-1 $\beta$ ) cytokines/chemokines and the immunoregulatory IL-10 cytokine. After subtracting the background values (Figure 1), a remarkably higher release of TNF- $\alpha$, a cytokine implicated in the pro-inflammatory pathway of monocyte activation and lower release of IL-10, a major anti-inflammatory cytokine, were observed in DS as compared to controls. Vaccine-specific release of IL-13, a cytokine implicated in the alternative pathway of monocyte activation and of IL-2, a cytokine implicated in the activation and homeostasis of conventional and regulatory $\mathrm{T}$ cells, were also lower in DS. Overall, the cytokine balance in response of PBMCs to the vaccine was shifted in favor of an inflammatory profile associated with defective IL-10 and IL-13 secretion in DS.

Adjuvants and/or components of whole pathogens contained in vaccines are required for vaccine immunogenicity. They act through activation of innate immune cells including monocytes. We have therefore analyzed vaccine-induced cytokine releases by monocytes. Results showed higher spontaneous release of IL-1 $\beta$ and IL- 6 by monocytes in DS than in $\mathrm{HC}$ (Table 1). In vitro stimulation of monocytes with the vaccine induced a significant increase over spontaneous release of IL-1 $\beta$, IL-6, and TNF- $\alpha$ in DS but of IL-10 in HC (Table 1). It is worth noting that in accordance with T-cell depletion, Th1, Th2, and Th17 cytokine responses were not induced by the vaccine in monocyte preparations. After subtracting spontaneous release, monocyte response to the vaccine in DS was clearly directed toward a pro-inflammatory (IL-1 $\beta$, IL-6, and TNF- $\alpha$ ) profile associated with defective counterregulatory IL-10 response (Figure 1). Monocytes are known to exist as polarized populations including M1 and M2 subsets. Although M1 monocytes/macrophages are powerful inflammatory cells that produce high levels of IL-1 $\beta$, IL- 6 , and TNF- $\alpha$ cytokines, M2 monocytes/macrophages produce low levels of these proinflammatory responses. ${ }^{12}$ Overall, our results support that monocytes/ macrophages are shifted in favor of a M1 proinflammatory phenotype in DS.

\section{4 | DISCUSSION}

Previous data supported a role for inflammation in epilepsy. ${ }^{6,8}$ Despite the clinical scenario of fever-induced and vaccine-induced seizure in DS, there are no data 


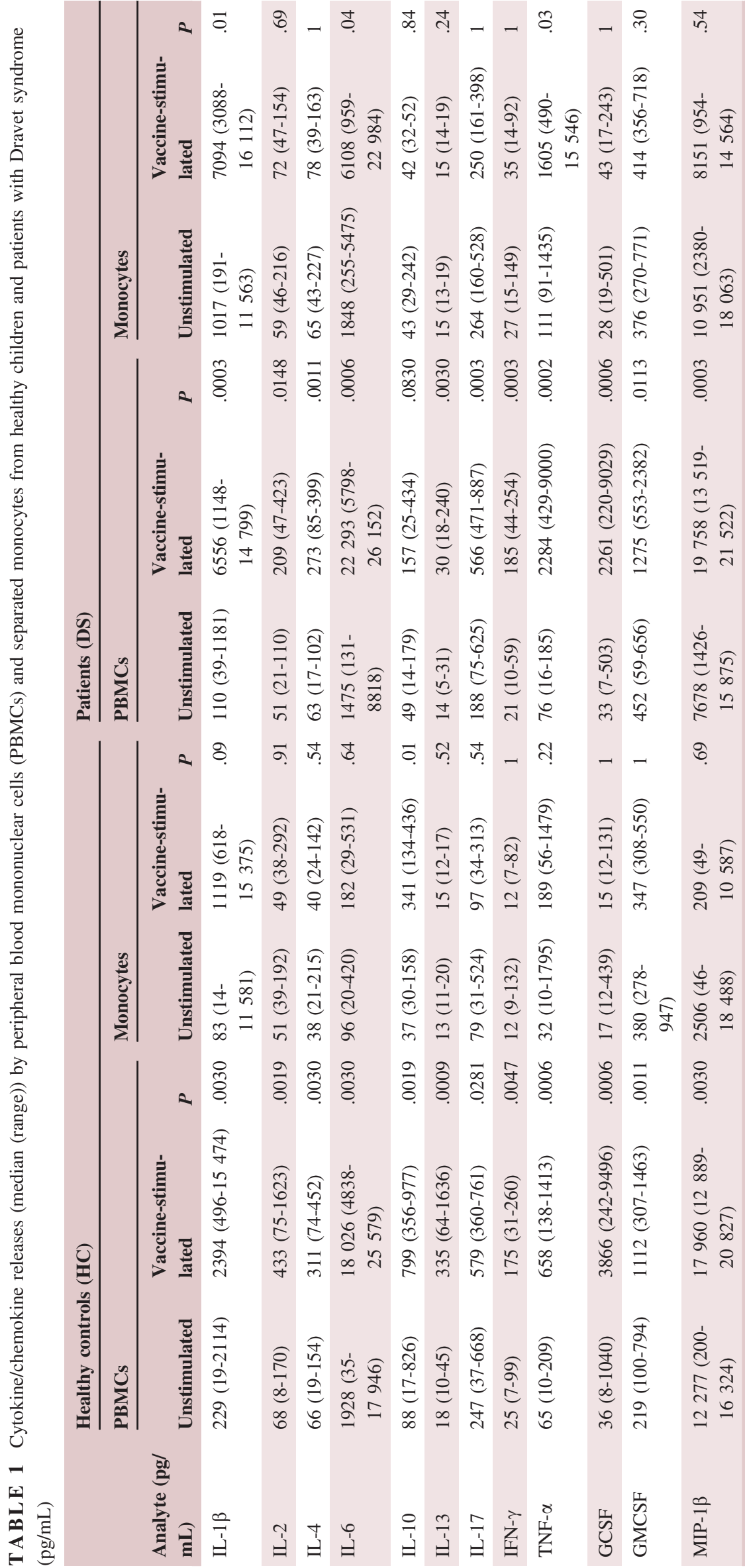




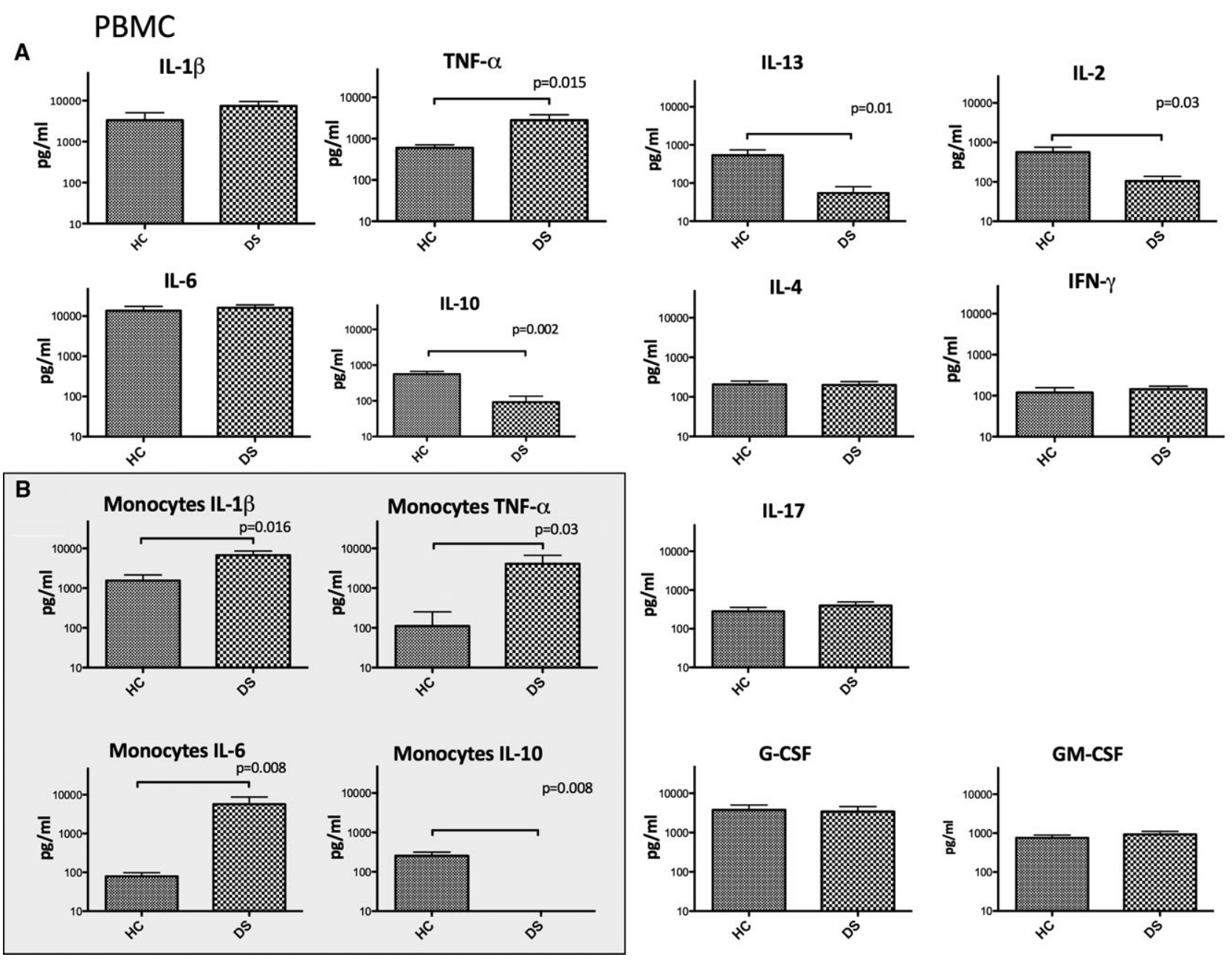

FIGURE 1 Comparison of vaccine-specific cytokine releases by immune cells from patients and from healthy children. The mean ( \pm SEM) concentrations $(\mathrm{pg} / \mathrm{mL})$ of vaccine-specific cytokine release (values observed in vaccine-stimulated cultures minus values observed in unstimulated cultures) by peripheral blood mononuclear cells (PBMCs, part A) and by monocytes (part B) are shown. The only cytokines with concentrations significantly more elevated in vaccine-stimulated than in vaccine-unstimulated cultures are illustrated. The Mann-Whitney test was used to compare results from patients with Dravet syndrome (DS) and healthy children (HC)l $P$ values are indicated when statistically significant $(P<.05)$

currently available on a possible alteration of the inflammatory response to vaccination in these patients. Our study provides the first evidence that vaccine responsiveness is biased toward a proinflammatory profile in DS.

There are accumulating data on the role of IL-1 $\beta$, IL-6, and high mobility group box 1 (HMGB1) in ictogenesis and epileptogenesis. ${ }^{7}$ In humans, immunohistochemistry studies of surgical samples of structural epilepsies are consistent with a role of these inflammatory mediators. ${ }^{6,7}$ Cytokine dosages in the blood of patients with epilepsy have been also done, but much methodological bias exists, as reported in a recent meta-analysis ${ }^{9}$ (timing of the blood sample, numerous epilepsy syndromes, and seizure types and frequency). Only IL-6 appears to be increased in the blood of patients with epilepsy, whereas IL-1 $\beta$ and TNF- $\alpha$ do not differ from controls. ${ }^{9}$
To avoid the bias described earlier, few studies have investigated cytokine secretion after in vitro stimulation of PBMCs. An increased amount of IL-1 $\beta$ (significant in 4/6), IL-6 (significant in 2/2), IL-10 (significant in 2/2), and IFN- $\alpha$ (significant in 1/1) has been found. ${ }^{9}$ PBMCs of patients with epilepsy and controls were stimulated with lipopolysaccharide (LPS) in 4 studies, ds-RNA of a common viral vector in one study, and mitogen in one study. ${ }^{9}$

In DS, we found a clear proinflammatory profile with an increase of IL- $1 \beta$, IL- 6 , TNF- $\alpha$, and a decrease of IL10 after in vitro vaccine stimulation of PBMCs. This profile is unique compared to previous studies that reported also an increase of the anti-inflammatory cytokine IL-10. ${ }^{9}$ The cytokine profile found in the monocytes of DS was comparable to the profile in PBMCs. Together, these results support a bias toward an M1 proinflammatory 
phenotype in DS at the expense of an M2 reparative and neuroprotective phenotype. Further studies are required to determine whether the discrepancies found in IL-10 level (decrease in our study and increase in past studies ${ }^{9}$ ) rely on the use of polyclonal activators in previous studies (recalled antigens in ours) or represent a selective downregulation of IL-10 in DS. It is notable that we also found lower vaccine-specific release by PBMCs of IL-13, a $\mathrm{T}$ cell-derived cytokine that drives monocyte differentiation toward an M2 phenotype. Altogether these results support for the first time that an altered interplay between the innate and the adaptive immune compartments occur in an epileptic syndrome. Whether the systemic changes we observed may reflect similar changes in brain monocytes/microglia remains to be addressed. Of interest sodium channel modulation contributes to effector roles of activated microglia. Pharmacological blockage of microglia reduced the phagocytic activity and proinflammatory cytokine release of microglia. ${ }^{13}$ The SCN1A mutation in DS might then be then a factor modulating the inflammatory properties of the monocytes.

Despite the small sample size, our results are sufficiently reliable to support a shift toward an M1 pro-inflammatory phenotype of monocytes in DS. Monocytes can adopt either M1 or M2 phenotypes. ${ }^{14}$ The activated M1 phenotype is predominately induced by an acute pathological condition such as stroke, traumatic brain injury, or experimentally induced systemic inflammation. ${ }^{14}$ M1 monocytes are involved in the production of proinflammatory cytokines, usually associated with negative consequences and that have been also described to promote ictogenesis and epileptogenesis. ${ }^{6}$ These results provide new insight into immune mechanisms associated with an epilepsy syndrome. Previous studies have already shown that peripheral inflammation can contribute to seizure precipitation or lower brain excitability. ${ }^{6}$ Our study may also guide research for the development of new immunotherapeutic agents in an epilepsy syndrome for which fever, viral illnesses, and immunization are frequent triggers of seizures during the whole course of the disease.

\section{ACKNOWLEDGMENTS}

This work was supported by INSERM, Université ParisDiderot, Fondation de l'Avenir.

\section{CONFLICT OF INTEREST}

Auvin reports personal fees for lectures or advice from BIOCODEX, EISAI, NOVARTIS, NUTRICIA, SHIRE, UCB Pharma, ULTRAGENYX, ZOGENYX, and GW PHARMA, outside the submitted work. None of the other authors have any conflict of interest to disclose. We confirm that we have read the Journal's position on issues involved in ethical publication and affirm that this report is consistent with those guidelines.

\section{AUTHOR CONTRIBUTIONS}

Stéphane Auvin: designed the study, collected patient samples, analyzed the data, wrote the manuscript; Mohamed Jeljeli: conducted the in vitro experiments, analyzed the data, edited the manuscript, Béatrice Desnous: collected clinical data, edited the manuscript; Nadia Soussi-Yanicostas: discussed the data and edited the manuscript; Pascal Dournaud: discussed the data and edited the manuscript; Ghislaine Sterkers: designed the study, supervised in vitro experiments, analyzed the data, wrote the manuscript.

\section{ORCID}

Stéphane Auvin (D) http://orcid.org/0000-0003-3874-9749

\section{REFERENCES}

1. Auvin S, Cilio MR, Vezzani A. Current understanding and neurobiology of epileptic encephalopathies. Neurobiol Dis. 2016;92:72-89.

2. Desnous B, Goujon E, Bellavoine V, et al. Perceptions of fever and fever management practices in parents of children with Dravet syndrome. Epilepsy Behav. 2011;21:446-8.

3. Tro-Baumann B, von Spiczak S, Lotte J, et al. A retrospective study of the relation between vaccination and occurrence of seizures in Dravet syndrome. Epilepsia. 2011;52:175-8.

4. von Spiczak S, Helbig I, Drechsel-Baeuerle U, et al. A retrospective population-based study on seizures related to childhood vaccination. Epilepsia. 2011;52:1506-12.

5. McIntosh AM, McMahon J, Dibbens LM, et al. Effects of vaccination on onset and outcome of Dravet syndrome: a retrospective study. Lancet Neurol. 2010;9:592-8.

6. Dupuis N, Auvin S. Inflammation and Epilepsy in the developing brain: clinical and experimental evidence. CNS Neurosci Ther. 2015;21:141-51.

7. Wilcox KS, Vezzani A. Does brain inflammation mediate pathological outcomes in Epilepsy? In Scharfman HE, Buckmaster PS, editors. Issues in clinical epileptology: a view from the bench. Berlin: Springer, 2014; p. 169-83.

8. Vezzani A, Aronica E, Mazarati A, et al. Epilepsy and brain inflammation. Exp Neurol. 2013;244:11-21.

9. de Vries EE, van den Munckhof B, Braun KPJ, et al. Inflammatory mediators in human epilepsy: a systematic review and metaanalysis. Neurosci Biobehav Rev. 2016;63:177-90.

10. Ohno Y, Ishihara S, Mashimo T, et al. Scn1a missense mutation causes limbic hyperexcitability and vulnerability to experimental febrile seizures. Neurobiol Dis. 2011;41:261-9.

11. Armand M, Chhor V, de Lauzanne A, et al. Cytokine responses to quantiferon peptides in pediatric tuberculosis: a pilot study. J Infect. 2014;68:62-70. 
12. Gordon S, Taylor PR. Monocyte and macrophage heterogeneity. Nat Rev Immunol. 2005;5:953-64.

13. Black JA, Liu S, Waxman SG. Sodium channel activity modulates multiple functions in microglia. Glia. 2009;57:1072-81.

14. Italiani P, Boraschi D. From monocytes to M1/M2 macrophages: phenotypical vs. functional differentiation. Front Immunol. 2014;5:514.

How to cite this article: Auvin S, Jeljeli M, Desnous B, Soussi-Yanicostas N, Dournaud P, Sterkers G. Altered vaccine-induced immunity in children with Dravet syndrome. Epilepsia. 2018;59:e45-e50. https://doi.org/10.1111/epi.14038

\section{SUPPORTING INFORMATION}

Additional Supporting Information may be found online in the supporting information tab for this article. 